\title{
Semantic category effects in visual word search
}

\author{
MARTIN B. KARLIN and GORDON H. BOWER \\ Stanford University, Stanford, California 94305
}

\begin{abstract}
The initial question was whether subjects could categorize a word semantically before they precisely identified the word itself. This failed to occur. When searching a visual display for a single target word, subjects searched at the same rate whether the distractors were in the same or in a different semantic category. However, when the size of the target set was increased to three, then six, items, subjects increasingly used category information to speed their search rate when targets and distractors belonged to different categories. Subjects appeared to perform the task by comparing the category of each display word to the category of the target set.
\end{abstract}

The question initiating these experiments was whether a reader would be able to categorize a word semantically before he could uniquely identify a word for what it was. We ask, for example, whether a reader can categorize the word green as a color term before he can identify it per se as the word green? The question sounds somewhat preposterous-after all, how could you look up a word's semantic category in your mental lexicon until after you had already looked up the word itself? Indeed, the question is preposterous if you think of mental classification as analogous to looking up the definition of a word in an actual dictionary.

However, this "dictionary look-up" idea could be fundamentally misleading. For example, there is some evidence that a person can classify an alphanumeric symbol as a number faster than he can identify it as a specific number, say 7. This conclusion has been supported in experiments by Egeth, Jonides, and Wall (1972) and Jonides and Gleitman (1972; see also Brand, 1971; Dick, 1971; Ingling, 1972; Nickerson, 1973). Egeth et al. had their subjects search through a visual field of symbols (letters or digits) to decide whether a target symbol was present. When the target was in the same category as the distractor symbols (e.g., both were letters or both were digits), time to reach a decision increased linearly with the number of elements in the display. However, when the target (say, a letter) was in a different category from the display distractors (say, digits), the decision time was independent of the display size. This was a most dramatic finding. More recent experiments have failed to replicate the finding that search time did not increase with increasing numbers of different-category items in the display (Gleitman,

The authors thank Arthur J. Flexser for his comments on an earlier version of this manuscript. Reprint requests may be sent to Gordon H. Bower, Department of Psychology, Stanford University, Stanford, California 94305.
Jonides, \& Friedman, Note 1; Jonides, Gleitman, \& Foland, Note 2). Rather, it has been found that the slope of the function relating reaction time (RT) to display size is considerably smaller for "different category" distractors than for "same category" distractors. Such results suggest either that classification sometimes occurs faster than identification of an alphanumeric character or that digits as a class may have certain visual features which distinguish them roughly from the class of letters (or vice versa).

The present studies explore whether a "category effect" can be produced with word stimuli where there is no possibility that the results would be explicable in terms of visual features distinguishing between words of different semantic classes. In scanning through a word list for the color word green, can people use category information to speed up their search when they know that the distractors in the display are names of trees rather than names of colors? If so, then one could conclude that semantic classification occurs at a very early stage in reading, perhaps before word identification.

Our experiments were analogous to those of Egeth et al. (1972) and Jonides and Gleitman (1972), with some differences. Their subjects were either "presence responders" or "absence responders." "Presence responders" had to press a key if the target was present, but made no response if it was absent. "Absence responders" pressed the key only if the target was absent. In our experiments, each subject responded "true" if the target was present and "false" if it was absent. Similarly, their subjects saw targets and distractors only in the same category or only in different categories, but never in both conditions. In our study, each subject saw targets in both the same and different categories as distractors in different blocks of trials.

The major procedural difference is in the presentation time of the stimuli. While Egeth et al. presented their alphanumeric symbols tachistoscopically 
Table 1

Exemplars of Stimuli for Various Conditions

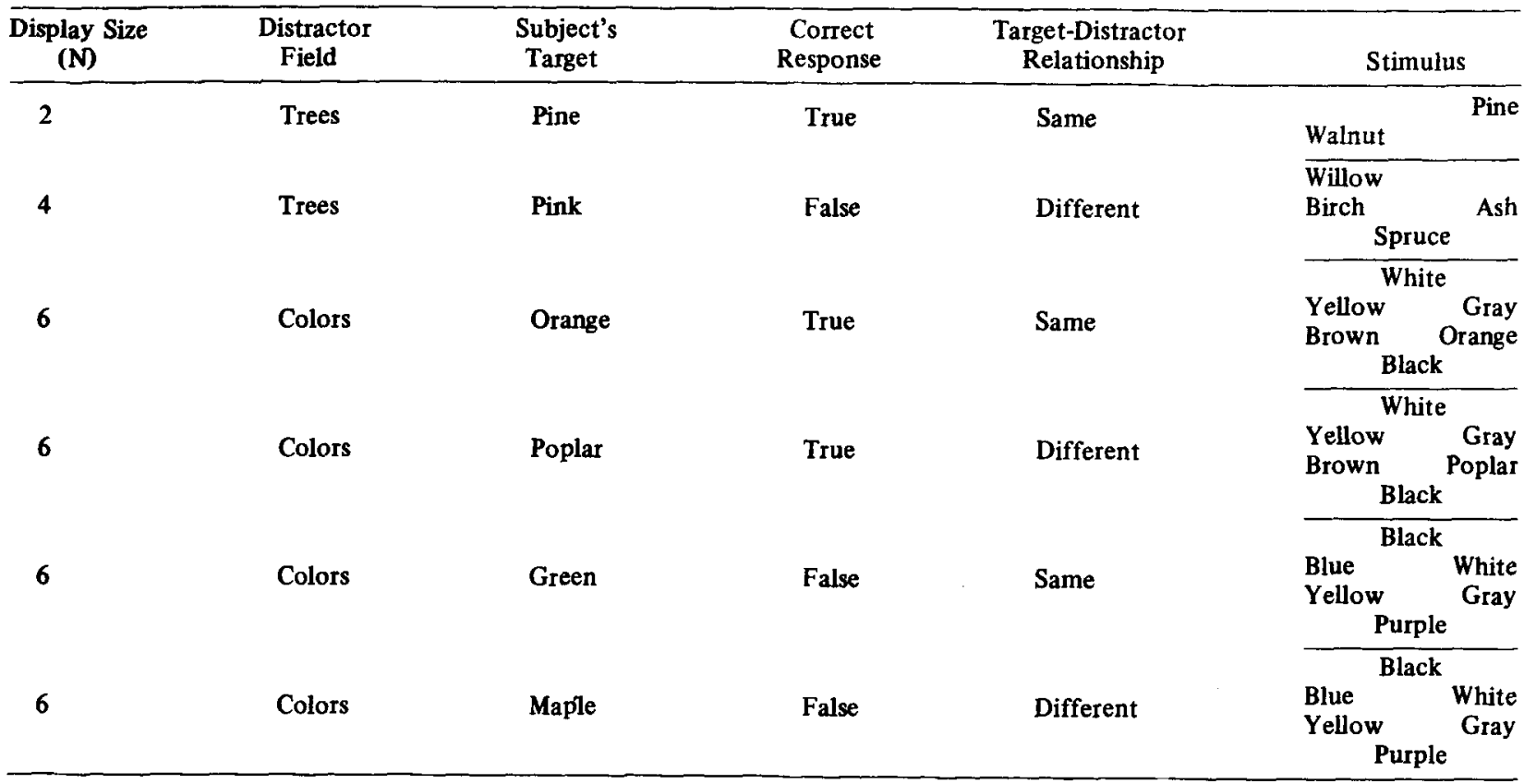

(on-time was $150 \mathrm{msec}$ ), stimuli in the present experiment remained in view until the subject responded. This procedure was called for since pilot work showed that subjects could not read arrays of two to six words accurately with such brief presentation times.

\section{EXPERIMENT I}

\section{Method}

Subjects. The subjects were 16 university students who were fulfilling a participation requirement for their introductory psychology course.

Design. Each subject viewed 216 slides. The stimuli were presented in two blocks of 108 slides each. In one block, the target and distractor items were from the same taxonomic category, while in the other block, they were from different categories. The order of presentation of the "same" and "different" blocks was counterbalanced across subjects. A target word was present in half of the slides in each block. The number of words on each slide could be two, four, or six. The category of the distractors for any particular subject remained constant for all slides viewed by that subject. Half the subjects always had color names as distractors, and the other half always had tree names.

Stimuli. Slides were prepared by typing each word in uppercase IBM Prestige type and then making 35-mm transparencies. Each slide contained two, four, or six words, which could appear at six possible locations on each slide. Table 1 illustrates some typical stimuli under various conditions. Note that the words in the second and third rows were always edge-justified, and those in the first and fourth rows were centered. If these six positions are conceptualized as points on a circle, at least two words on each slide appeared at diametrically opposed loci. Each word and each location was used equally often, and any particular target word appeared equally often in each location.
Words were selected from two categories in the Battig and Montague (1969) norms, Colors and Trees. Twelve of the most frequently produced words having from three to six letters were selected from each category. Of these, three in each category were selected as possible target words. (For colors, these were green, orange, and pink; for trees, they were maple, poplar, and pine.) The remaining nine words of each category were used equally often as distractors.

Stimuli of the appropriate display size were generated randomly within the constraints mentioned above. Only one target word, if any, appeared on a slide, and no word appeared twice on any slide. Table 1 illustrates some of the properties of the stimuli in various conditions. There were four types of slides. True-same slides had a target present in the same category as the distractors. True-different slides had a target present from the different category. Table 1 reveals the interesting relationship between the two types of "true" slides. Each truedifferent slide had a true-same mate which was identical, except that a new target word, in the same category as the distractors, was substituted for the old "different" target. The distractor items on the two mates remained unchanged.

False-same slides had no target present, and the subject was searching for targets in the same category as the distractors. False-different slides had no target, but the subject was searching for targets in a different category from the distractors. As illustrated in Table 1 , the set of false-same slides was identical to the set of false-different slides; the only difference was the target for which the subject was searching.

Stimuli were ordered randomly within each of the four blocks of trials (tree targets among color distractors, color targets among colors, tree targets among trees, and color targets among trees). All subjects saw the slides within each block in the same order, each subject viewing only two of the possible four blocks (those with identical distractor fields).

Subjects were seated about $9 \mathrm{ft}$ from the projected images, which covered an area of about $5 \times 7$ in. The slides thus subtended a visual angle of about $2.5^{\circ}$ vertically and $3.5^{\circ}$ horizontally. 
Table 2

Summary Statistics for Experiments I, II, and III*

\begin{tabular}{clcr}
\hline Experiment & Condition & Slope \pm SE (msec/item) & Intercept \pm SE (msec) \\
\hline 1 & False-Different & $124 \pm 7.4$ & $432 \pm 24.0$ \\
& False-Same & $127 \pm 8.3$ & $406 \pm 21.4$ \\
& True-Different & $68 \pm 4.8$ & $516 \pm 35.1$ \\
2 & True-Same & $68 \pm 5.8$ & $499 \pm 27.3$ \\
& False-Different & $191 \pm 12.9$ & $437 \pm 40.0$ \\
& False-Same & $248 \pm 19.3$ & $375 \pm 41.0$ \\
3 & True-Different & $102 \pm 8.0$ & $547 \pm 31.1$ \\
& True-Same & $117 \pm 8.4$ & $544 \pm 24.3$ \\
& False-Different & $159 \pm 18.0$ & $413 \pm 27.6$ \\
& False-Same & $272 \pm 21.2$ & $385 \pm 42.6$ \\
\hline
\end{tabular}

"Note: Slopes, intercepts, and their corresponding standard errors are based on individual regression analyses by subject.

Procedure. Subjects were first told to familiarize themselves with two lists which contained all possible colors and trees used in the experiment. They were told the general nature of the task, and were told to respond "as quickly and as accurately as possible." After being told the possible target and distractor words for the current block of stimuli, the subjects responded to 18 practice slides. Then the first block of 108 trials was presented, after which subjects were told the new possible target words, saw more practice slides, and proceeded with the second block of trials.

Each trial of the experiment can be described as follows. Subjects were instructed to fixate at the middle of a small display screen at the start of each trial. A "ready" cue at the beginning of a trial was signaled by the changing of a Kodak Carousel projector. There was a 2-sec pause during which the experimenter read aloud the appropriate target word for that trial (e.g., "PINE"). Then a Lafayette electronic shutter opened, displaying the slide, and a Hunter Klockounter started. The slide remained on until the subject responded by pressing one of two thumb switches, at which point the shutter closed, the clock stopped, and the subject's response was indicated on a pilot light. The experimenter recorded RT and the subject's response. No feedback was given the subject. Seven seconds after the original ready cue, the next trial began.

\section{Results and Discussion}

In all three experiments to be reported, analyses of variance showed that it made no difference whether subjects first saw the "same" distractors or the "different" distractors block. Also, subjects who saw color names as distractors did not differ from subjects who saw tree names as distractors. Therefore, statistics were pooled across both of these factors.

For each subject, four lines relating RT to display size $(2,4$, or 6$)$ were derived corresponding to the four classes of stimuli (trials) described above (true-same, true-different, false-same, and falsedifferent). Each line was described by its slope and intercept on the RT axis. These individual slopes and intercepts were compared using the method of orthogonal contrasts.

The results of Experiment I appear at the top of Table 2. Figure 1 shows the relationship between RT and display size, pooled over subjects, with associated error rates for each point. The lines drawn through the empirical data points are theoretical curves predicted by a model to be described later.

Figure 1 shows the main effects supported by the statistical analysis. True stimuli were processed faster than false stimuli, $t(15)=10.2, p<.01$. The empirically derived curves had a significant linear component for both trues $[t(15)=15.6, p<.01]$ and falses $[t(15)=17.9, p<.01]$. The false curves had a significant nonlinear (quadratic) component $[t(15)=2.5, p<.05]$, but the "true" curves did not $[t(15)=1.4$, n.s. $]$. The intercepts of the true-same and true-different curves did not differ $[t(15)=0.7$, n.s.], nor did the intercepts of the false-same and false-different curves $[t(15)=1.4$, in.s.].

The comparisons of greatest interest involve the slopes for the "same" stimuli vs. the "different" stimuli. We examined these separately for trues and

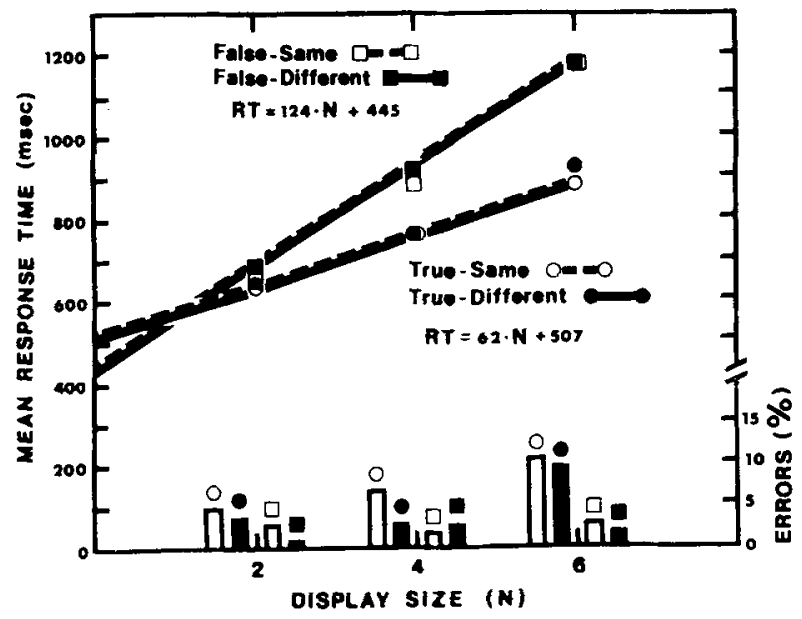

Figure 1. Error rate and mean response time as a function of display size for a one-element target set; Experiment $I$. 


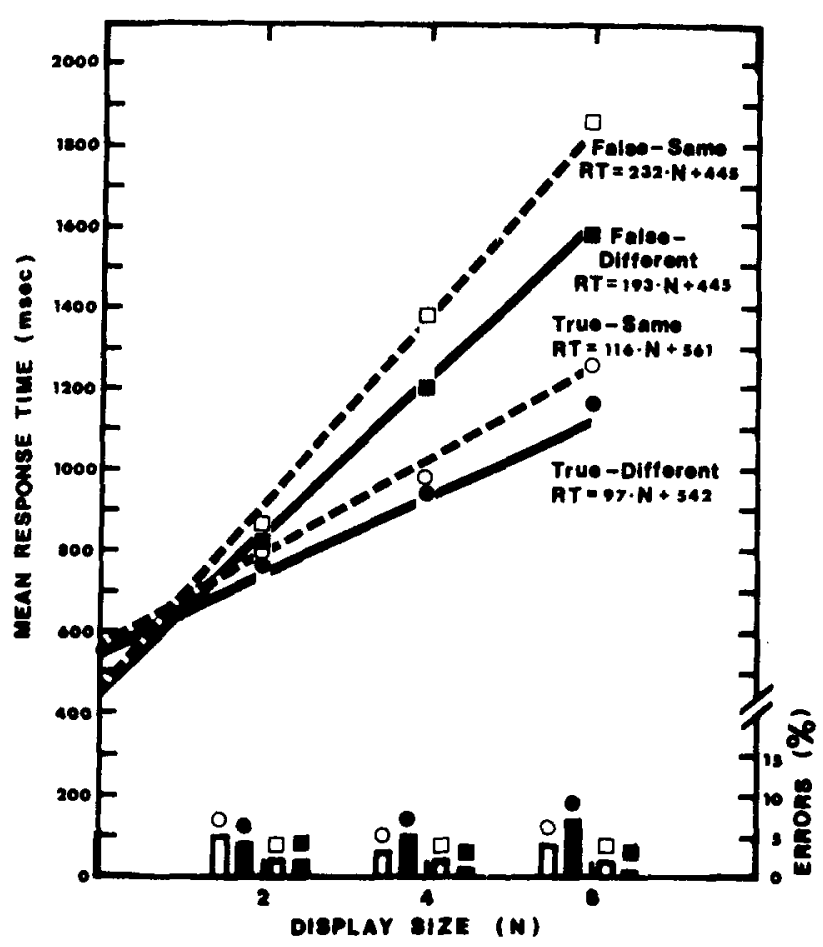

Figure 2. Error rate and mean response time as a function of display size for a three-element target set; Experiment II.

falses. Egeth et al. had found that slopes for sames were greater than slopes for differents for both presence and absence responders (corresponding to our trues and falses). Table 2 shows that the slopes of the true-same and true-different curves were virtually identical, as were the slopes of the falsesame and false-different curves, with $t(15)=0.04$ for trues and $t(15)=0.4$ for falses.

Basically, we have failed to find the "category effect" we sought: subjects searched for the word green through other color names just as rapidly as through tree names. Why might we fail to get the category effect with words when it was found with digits and letters? One possibility is that word categorization just follows word identification, and the early digit-letter results are explicable in terms of visual features distinguishing those two classes. Another possibility, suggested by many of our subjects' introspections, is that our subjects did not have to process the stimuli as words at all, since they could perform the task rapidly by examining only physical features of the words without ever "reading.",

As an illustration, suppose the target word for a trial were maple. Then the subject could set up a test to look for an " $\mathrm{m}$ " in the initial position or a medium-length word with an overall shape having a "hump" near the end (the letter l). Any candidate word failing such a criterion would be immediately rejected, whereas candidates passing such a criterion would be examined more closely for more matching features. In any event, if subjects were using only physical features or fragments, then in a real sense we have not gotten the subject to make the decision at the level of interest to our project.

We asked, how can we make it more likely that the person will read the full word while scanning rather than simply checking for a physical feature from the target word? By increasing the number of possible target words that could be in the display, we hoped to decrease the efficiency of a simple physical-feature-checking strategy and increase the likelihood that subjects would process the whole word. Accordingly, in Experiment II, subjects searched for one of three target words that belonged to the same semantic category.

\section{EXPERIMENT II}

\section{Method}

Sixteen more subjects were drawn from the same source. All other aspects of the experiment remained the same, except that the experimenter did not read the appropriate possible target prior to each trial. Rather, subjects were told, at the beginning of each block of 108 trials, the three possible target words that could appear on each trial. Either zero or one target word appeared on every trial, the three different target items appearing equally often over trials.

\section{Results and Discussion}

Figure 2 shows the relationship between RT and display size, pooled over subjects. Recall that the curves represent theoretical fits of the process model. Summary statistics appear in the middle section of Table 2.

The statistic of major interest compared the slopes for "same" and "different" conditions. The falsesame line had a larger slope than the falsedifferent line, $t(15)=5.0, p<.01$. The true-same slope appears to be larger than the true-different slope, but this difference is not quite statistically significant, $t(15)=1.86, p<.10$.

The other comparisons are quite similar in pattern to those of Experiment I (all $d f=15, p<.01$ ). "True" stimuli are processed more quickly than "false" stimuli ( $t=9.8)$. Both "true" and "false" stimuli had significant linear components ( $t=15.3$ and 14.3 , respectively), while significant quadratic components were obtained for trues $(t=4.0)$, but not for falses $(t=1.0$, n.s. $)$. True-same and true-different intercepts did not differ $(t=0.1$, n.s.), nor did false-same and falsedifferent intercepts $(t=2.0, p<.10)$.

The pattern of results indicates that subjects appear to be processing the stimuli semantically, since the relationship of targets to distractors 
is affecting search rate significantly, particularly for "false" stimuli. By further increasing the size of the possible target set to six, we hoped to amplify this "category effect," since searching for one of six targets among distractor items in the same category should become even more difficult compared to searching through distractors in the different category.

\section{EXPERIMENT III}

\section{Method}

Sixteen additional subjects from the introductory psychology pool participated in the third experiment. Stimuli were changed so that there were six possible target words instead of three. Three additional words were selected from the original set of norms (colors: maroon, gold, indigo; trees: beech, apple, cherry). Then half of the "true" slides for each block of trials were changed by substituting one of the new targets for an old target. In Experiment III, subjects were told that any one of six words could appear as targets. All other aspects of the experiment remained the same.

\section{Results and Discussion}

Results for this experiment are plotted in Figure 3 and reported at the bottom of Table 2 . Significant differences (all $d f=15, p<.01$ ) were obtained as follows: Trues were faster than falses $(t=8.0)$, linear slopes were obtained for both trues $(t=12.8)$ and falses $(t=12.3)$, while quadratic components were nowhere significant (for trues, $t=1.8$, n.s.; for falses, $t=1.2$, n.s.). Of major interest is that, in this experiment, "same" slopes were greater than "different" slopes for both trues $(t=3.7)$ and falses $(t=6.2)$. Intercepts were equal for sames vs. differents in trues $(t=1.7$, n.s. $)$ and falses $(t=0.8$, n.s. $)$.

Subjects in this experiment reported that "different" targets were easier to find than "same" targets, and that they were using category information during the search.

The data show two striking patterns. First is the general linearity of the display-size effect. Second, within given field conditions, the slope for "false" RTs was always about twice the slope for "true" RTs. Sternberg (1969) suggested that a serial self-terminating search process implies such a 2:1 slope relationship. On the other hand, the frequent empirical result of equal slopes for positive and negative probes in memory scanning has been taken as evidence for an exhaustive search. The present study involves visual scanning, and the evidence regarding slopes on positive and negative trials of visual scanning has been inconsistent. Some early visual scanning studies by Nickerson (1966) and Sternberg (Note 3) obtained slope differences (although not of a 2:1 magnitude), whereas equal slopes were reported by Atkinson, Holmgren, and Juola (1969), Egeth et al. (1972), and Jonides

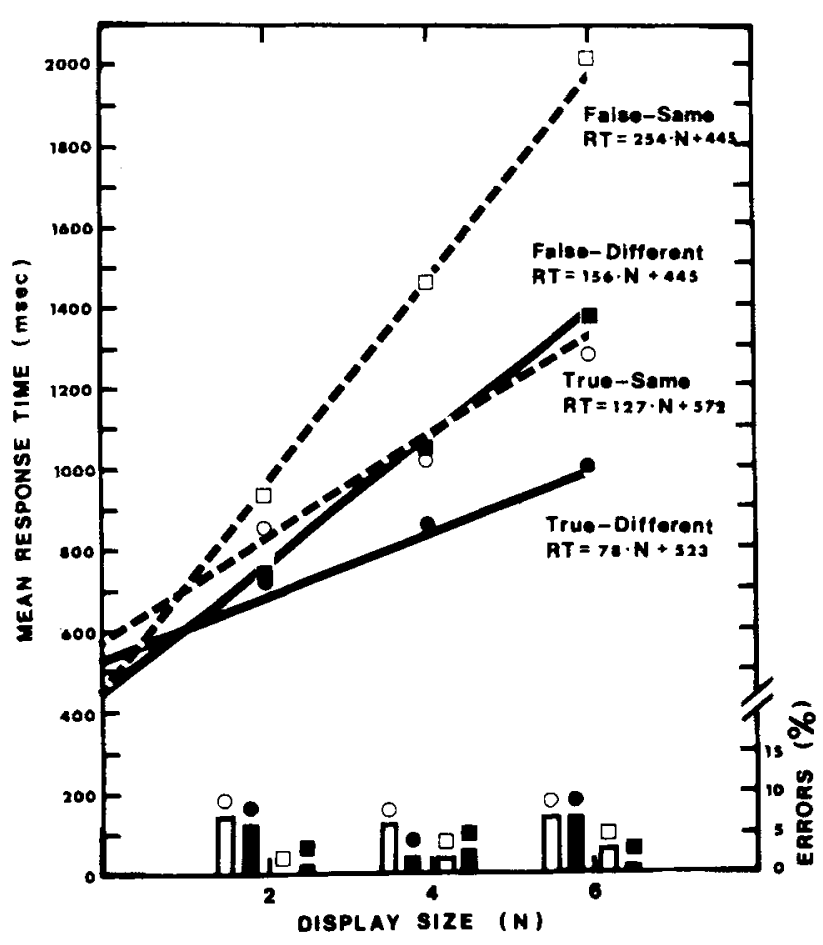

Figure 3. Error rate and mean response time as a function of display size for a six-element target set; Experiment 11 .

and Gleitman (1972). A critical variable for the 2:1 slope difference may be whether or not eye movements are required to scan the entire visual array. In our experiment, such eye movements were clearly required, despite efforts to compact the words around the fixation point.

A process model. We will present a simple process model which economically describes our data. It is but one of a fairly large class of nearly equivalent models, so we make no claims regarding the accuracy of its details. The principal assumptions are that the subject executes a serial self-terminating scan over the display items; in certain field conditions, as each item is scanned, he reads and categorizes it as a tree or as a color; in other conditions, he reads and then compares it to his target set in memory by an exhaustive scan.

Recall that the "same" field or "different" field conditions occur separately in large blocks of trials. In the "same" field condition, categorization is useless and the subject must compare each display word to the targets in memory. However, in the "different" field condition, it would be possible for the subject to set himself to follow a "categorization strategy" if it is to his advantage. We suppose that categorization of a "different" field item becomes more preferred to searching the memory set for its mate the longer the time required for the memory search. This memory search time will increase as the target set increases from one to 


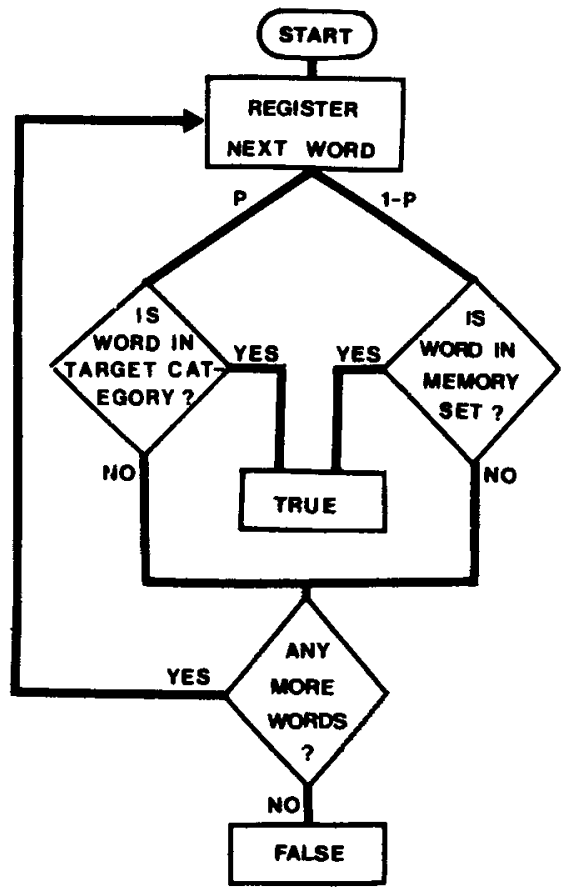

Figure 4. Flowchart of the process model.

three to six (across our three experiments). Since the advantage of using the categorization strategy in the "different" field condition varies with the three target-set sizes, we introduce an arbitrary parameter, $P$, to denote the probability that a subject uses a categorization strategy rather than a memorysearch strategy during the "different" field condition.

Figure 4 depicts a composite flow diagram for the model. All the conditions can be handled by specialization of this one model. At onset of the $\mathrm{N}$-element display, the model begins scanning through the display, reading one word at a time, terminating as soon as a "true" or "false" decision can be reached. Consider first the block of "same" field trials. Here categorization is useless; the task can be done only by comparing the candidate field item to the target set in memory. Thus, for "same" field trials, the right-hand branch will always be taken (set $P=0$ ). The time required for the exhaustive comparison of the candidate field item to the memory set will be $m_{1}, m_{3}$, or $m_{6}$ for target sets of size one, three, or six. This process leads to the equations in Table 3 for the "same" field conditions for "true" and "false" trials. The additive constant $K$ is included to indicate initial preprocessing time and the time to execute the response. It is the same across all conditions.

Next consider "different" field trials. Here, if memory search requires a lot of time, then categorization may be more efficient. (Recall that for "different" field trials, if any field item belonged to the target category, then it was indeed one of the target items.) Let $c$ denote the time required to read a candidate word, categorize it, and compare its category to the target category. With $i$ target items, the processing time per item is then either $c$ or $m$. The average processing time is the probabilistic mixture of $c$ and $m_{i}$, viz,

$$
\text { average rate }=P c+(1-P) m_{i} .
$$

Applied to a self-terminating search process, this processing rate leads to the true-different and falsedifferent equations in Table 3.

It turns out that the data are well approximated by assuming that categorization is always done $(P=1)$ when there are six targets, that memory-set searching is always done $(1-P$ $=1$ ) when there is only one target, and that categorization or memory searching are done about equally often $(P=.50)$ when there are three targets. These values for $P$ are shown along with the equations in Table 3 . The parameter, $P$, may be thought of in two ways. First, it could be the probability that the subject consciously chooses to follow the category vs. the memory-search strategy, the choice being made either on each trial or for an entire block of trials. Second, the memory-search and category decision processes with respect to a display word could be activated in parallel and the decision made as soon as either one is completed (a "race" model of RT). In this view, $P$ would be the probability that the category decision becomes available before the memory-search decision.

Table 3

Predicted Equations of the Model

\begin{tabular}{|c|c|}
\hline \multicolumn{2}{|c|}{ Experiment $\mathbf{I}$ (target set size $=1$, set $P$ to 0 ) } \\
\hline False-Different & $\begin{aligned} \mathrm{RT} & =\mathrm{K}+\mathrm{N} \cdot\left[(0 \cdot \mathrm{c})+(1-0) \cdot \mathrm{m}_{1}\right] \\
& =\mathrm{K}+\mathrm{N} \cdot \mathrm{m}_{1}\end{aligned}$ \\
\hline $\begin{array}{l}\text { False-Same } \\
\text { True-Different }\end{array}$ & $\begin{aligned} \mathrm{RT} & =\mathrm{K}+\mathrm{N} \cdot \mathrm{m}_{1} \\
\mathrm{RT} & =\mathrm{K}+[(\mathrm{N}+1) / 2] \cdot\left[(0 \cdot \mathrm{c})+(1-0) \cdot \mathrm{m}_{1}\right] \\
& =\mathrm{K}+[(\mathrm{N}+1) / 2] \cdot \mathrm{m}_{1}\end{aligned}$ \\
\hline True-Same & $\mathrm{RT}=\mathrm{K}+[(\mathrm{N}+1) / 2] \cdot \mathrm{m}_{1}$ \\
\hline \multicolumn{2}{|c|}{ Experiment II (target set size $=3$, set $P$ to .5 ) } \\
\hline $\begin{array}{l}\text { False-Different } \\
\text { False-Same } \\
\text { True-Different } \\
\text { True-Same }\end{array}$ & $\begin{array}{l}\left.R T=K+N \cdot[P \cdot c)+(1-P) \cdot m_{3}\right] \\
R T=K+N \cdot m_{3} \\
R T=K+[(N+1) / 2] \cdot\left[(P \cdot c)+(1-P) \cdot m_{3}\right] \\
R T=K+[(N+1) / 2] \cdot m_{3}\end{array}$ \\
\hline \multicolumn{2}{|c|}{ Experiment III (target set size $=6$, set $P$ to 1 ) } \\
\hline False-Different & $\begin{aligned} \mathrm{RT} & =\mathrm{K}+\mathrm{N} \cdot\left[(1 \cdot \mathrm{c})+(1-1) \cdot \mathrm{m}_{6}\right] \\
& =\mathrm{K}+\mathrm{N} \cdot \mathrm{c}\end{aligned}$ \\
\hline $\begin{array}{l}\text { False-Same } \\
\text { True-Different }\end{array}$ & $\begin{aligned} \mathbf{R T} & =\mathrm{K}+\mathrm{N} \cdot \mathrm{m}_{6} \\
\mathbf{R T} & =\mathrm{K}+\left[(\mathrm{N}+1) / 21 \cdot\left[(1 \cdot \mathrm{c})+(1-1) \cdot \mathrm{m}_{6}\right]\right. \\
& =\mathrm{K}+[(\mathrm{N}+1) / 2] \cdot \mathrm{c}\end{aligned}$ \\
\hline True-S & $\mathrm{RT}=\mathrm{K}+[(\mathrm{N}+1) / 2] \cdot \mathrm{m}_{6}$ \\
\hline
\end{tabular}


Fitting the model. A least squares estimation program was used to fit the model to the data in Figures 1, 2, and 3 . There are 36 points to be described by six parameters- $P, c, m_{1}, m_{3}$, $m_{6}$, and $K$. The model fits are drawn in Figures 1,2 , and 3 , and are seen to be fairly close, with a root mean squared deviation of $29 \mathrm{msec}$. The parameter estimates were: $K=445 \mathrm{msec}, c=156 \mathrm{msec}$, $m_{1}=124 \mathrm{msec}, m_{3}=232 \mathrm{msec}, m_{6}=254 \mathrm{msec}$, and $P=0,0.5$, or 1 for target sets of sizes 1,3 , and 6 .

We will note several features of these parameters. First, in Experiment $I$, the memory search parameter, $m_{1}$, is less than the categorization time, $c$, so it is more efficient to always carry out memory searches; on the other hand, in Experiment III the memory search parameter, $m_{6}$, is considerably larger than the categorization time, so it is more efficient to do the task by categorization. Second, the values of $m$, memory-set search time, do not increase linearly with the set size. This could be because the comparison process for the singletarget set of Experiment I involved a match of physical fragments which differed fundamentally from the process with three or six targets.

\section{GENERAL DISCUSSION}

Our results on visual searching may be compared to those of Naus, Glucksberg, and Ornstein (1972) and Naus (1974), who investigated memory scanning. In their experiments, subjects searched for a target through memorized lists containing words from one to four semantic categories. Their results suggested that subjects used category information during their search. Instead of searching an entire multiple-category list exhaustively, subjects stopped their memory scanning after they had searched through the category of the test item. Further, Naus (1974) showed that subjects could alter their search strategy to meet task demands. These results lend support to our findings on visual searching.

Do our results shed any light on the findings of Egeth et al. (1972) and Jonides and Gleitman (1972)? Yes, but a small chain of arguments is required. We have shown that a category effect can be obtained (for "different" field conditions) whenever the time to read and categorize an item is faster than the time to read and compare an item to a target set in memory. How might a category effect be obtained with a one-element target with the alphanumeric stimuli of Egeth et al. but not with our words? We know that people can search memory for words just as fast as for alphanumeric symbols (see Clifton \& Tash, 1973; Sternberg, 1975), so a difference in that respect could not be causing the differing outcomes. Perhaps, then, categorization of alphanumeric symbols (into letters vs. digits) is faster than semantic categorization of words (into trees vs. color names). We checked this possibility in a brief subsidiary experiment.

We had seven college subjects classify two 18 -item lists. One list contained in random order nine of the uppercase letters used by Jonides and Gleitman (C, E, F, H, J, P, R, U, Y) and the nine digits, 1-9. The other list contained nine of the color names and nine of the tree names used in our experiments. We measured RT to classify the alphanumerics into digits vs. letters and the word list into trees vs. colors, counterbalancing the order of the two lists. The mean classification RT was $490 \mathrm{msec}$ for the alphanumerics and $542 \mathrm{msec}$ for the words. This difference is significant, $t(6)=3.12$, $p<.05$. Assuming that the reading time for alphanumerics is the same as reading time for words (see Woodworth, 1940), the 52-msec difference found above suggests that the letter-digit classification is faster than the trees-colors classification. This is probably due to the fact that the letter-digit discrimination is overly familiar and may be based on class-distinguishing visual features. In any event, this faster categorization time for alphanumerics would, according to our model, tend to produce a "category effect" for alphanumerics even with one-element target sets, as was found by Egeth et al.

Our findings do not resolve the issue of whether categorization can precede identification in these tasks. For example, the descriptive model is simply indifferent to this issue, since its categorization or memory-search processes could in principle be initiated either before or after the scanned word was identified (named). In retrospect, the question whether identification precedes classification in general seems poorly formulated. We can all easily think of examples where identification precedes classification (e.g., saying 7 is "seven" before classifying it as "a prime number"), as well as contrary cases where classification precedes identification (e.g., saying "airplane" before "DC-747," or saying "dog" before "Irish Setter"). Identification will precede classification when the classification is not based on visual similarities among the class of objects but is given by enumeration (e.g., consonants vs. vowels, odd vs. even numbers, etc.). Classification will precede identification when judgments about the more general category requires noticing fewer physical features of the stimulus than do judgments about the subordinate category. With this view, then, particular outcomes comparing speeded judgments of superordinate vs. subordinate 
categories are to be analyzed in their own right. Thus, for example, we should be asking of the Egeth et al. findings: What are the physical features that probably distinguish digits as a class from letters as a class? Further experiments should be performed to explore this question in greater detail.

In conclusion, there appears to be a processing tradeoff involving memory load and the ease of the category decision. The presence or absence of the so-called "category effect" results from an interaction between the type of stimulus and the definition of the category decision for a particular task.

\section{REFERENCE NOTES}

1. Gleitman, H., Jonides, J., \& Friedman, R. B. The cost of categorization in visual search: Incomplete processing of target and field items. Manuscript submitted for publication, 1975.

2. Jonides. J.. Gleitman. H., \& Foland, E. The benefit of categorization in visual search: Target location without identification. Manuscript submitted for publication. 1975.

3. Sternberg. S. Scanning a persisting visual image versus scanning a memorized list. Paper presented at the annual meeting of the Eastern Psychological Association. April 1967. Boston.

\section{REFERENCES}

Atrinson. R. C.. Holmgren. J. E., \& Juola, J. F. Processing time as influenced by the number of elements in a visual display. Perception \& Psychophysics, 1969. 6. 321-326.

Batrig, W. F., \& Montague. W. E. Category norms for verbal items in 56 categories: A replication and extension of the Connecticut Category Norms. Journal of Experimental Psychology Monograph, $1969.80 \times 3$. Pt. 2).

Brand, J. Classification without identification in visual search.
Quarterly Journal of Experimental Psychology, 1971, 23. 178-186.

Clifton, C.. JR.. \& TASH. J. Effect of syllabic word length on memory search rate. Joumal of Experimental Psychology. 1973. 99. 231-235.

Dick, A. O. Processing time for naming and categorization of letters and numbers. Perception \& Psychophysics. 1971. 9. 350-352.

Egeth. H. E.. Jonides. J.. \& Wall, S. Parallel processing of multielement arrays. Cognitive Psychology, 1972, 3. 674-698.

INGLING, N. W. Categorization: A mechanism for rapid information processing. Journal of Experimental Psychology. 1972. 94. 239-243.

Jonides. J.. \& Gleitman. H. A conceptual category effect in visual search: $O$ as letter or digit. Perception \& Psychophysics, 1972. 12, 457-460.

NAUS. M. J. Memory search of categorized lists: A consideration of alternative selt-terminating search strategies. Journal of Experimental Psycholog:. 1974. 102. 992-1000.

Naus. M. J.. Glucksberg. S., \& Ornstein, P. A. Taxonomic word categories and memory search. Cognitive Psychology. 1972, 3, 643-654.

Nickerson. R. S. Response times with a memory-dependent decision task. Joumal of Experimental Psychology, 1966. 72. $761-769$.

Nickerson. R. S. Can characters be classified directly as digits vs letters or must they be identified first? Memory \& Cognition. 1973. 1. 477-484.

Sternaerg. S. Memory-scanning: Mental processes revealed by reaction-time experiments. American Scientist, 1969. 57. $421-457$.

Sternberg. S. Memory scanning: New findings and current controversies. Quarterly Joumal of Experimental Psychology. 1975, 27, 1-32.

WOODwORTh, R. S. Psychology (4th ed.). New York: Holt, 1940. P. 40.

(Received for publication October 3, 1975; revision received January $30,1976$. 